\title{
Proton beams generated with high-intensity lasers: Applications to medical isotope production
}

\author{
S. Fritzler, V. Malka, ${ }^{\text {a) }}$ G. Grillon, J. P. Rousseau, and F. Burgy \\ Laboratoire d'Optique Appliquée-ENSTA, UMR 7639, CNRS, École Polytechnique, \\ 91761 Palaiseau, France \\ E. Lefebvre and E. d'Humières \\ Département de Physique Théorique et Appliquée, CEA/DAM Ile-de-France, BP 12, \\ 91680 Bruyères-le-Châtel, France \\ P. McKenna and K. W. D. Ledingham ${ }^{\text {b) }}$ \\ Department of Physics, University of Strathclyde, Glasgow G4 OGN, United Kingdom
}

(Received 5 March 2003; accepted 13 August 2003)

\begin{abstract}
Proton beams of up to $10 \mathrm{MeV}$ have been obtained by the interaction of a $10 \mathrm{~Hz}$ "table-top" laser, focused to intensities of $6 \times 10^{19} \mathrm{~W} / \mathrm{cm}^{2}$, with 6 - $\mu \mathrm{m}$-thin foil targets. Such proton beams can be used to induce ${ }^{11} \mathrm{~B}(p, n){ }^{11} \mathrm{C}$ reactions, which could yield an integrated activity of $13.4 \mathrm{MBq}(0.36$ $\mathrm{mCi}$ ) after $30 \mathrm{~min}$ laser irradiation. This can be extended to $\mathrm{GBq}$ levels using similar lasers with kilohertz repetition rates, making this positron-emission tomography isotope production scheme comparable to the one using conventional accelerators. () 2003 American Institute of Physics.

[DOI: $10.1063 / 1.1616661]$
\end{abstract}

The production of short-lived isotopes such as ${ }^{11} \mathrm{C}$ or ${ }^{18} \mathrm{~F}$ is important in medicine for positron-emission tomography (PET), since they undergo $\beta^{+}$decays with half-lives of 20.4 and $109.7 \mathrm{~min}$, respectively. When these positrons pass through matter, they slow down and annihilate at rest with free electrons, which produces two counterpropagating photons of $511 \mathrm{keV}$. Using an arrangement of coincidence detectors at $180^{\circ}$ around a sample with such a radioactive probe inside, the activity and location of the radioisotopes can be determined. This principle is used in medical imaging, where these radiopharmaceuticals are injected into a patient. The radio-isotope carriers are then taken up wherever they are used in the body, enabling the imaging of blood flow or brain tumors. Nowadays, these radiopharmaceuticals are generated using proton beams produced by cyclotrons or Van de Graaff accelerators, which induce $(p, n)$ reactions with ${ }^{11} \mathrm{~B}$ and ${ }^{18} \mathrm{O}$ nuclei inside a target. Due to the size, cost, and shielding required for such installations, PET is limited to only a few facilities.

In the last few years, it has been demonstrated that laser-plasma interactions can accelerate protons to energies up to $58 \mathrm{MeV}$. ${ }^{1}$ A laser beam focused to high intensity onto solid targets creates a plasma. In this plasma, the laser accelerates electrons, which propagate through the target and set up a space-charge field when they exit. Due to this strong static-electric field, ions can get accelerated perpendicularly to the target surface.

Even though the energy spectrum of these accelerated protons is presently a broad Maxwellian-like distribution, they can nevertheless be used for the generation of radioisotopes as long as their energy is greater than the $Q$-value; that is, the threshold of the $(p, n)$ reaction and the number of

\footnotetext{
${ }^{a)}$ Electronic mail: victor.malka@ensta.fr

${ }^{b)}$ Also at: AWE plc, Aldermaston, Reading RG7 4PR, UK.
}

protons is sufficient to obtain an appropriate activation. Initially, such nuclear reactions were implemented to obtain the yield of laser-produced protons. However, as their flux can be important, high-intensity lasers were considered as an alternative method for PET isotope production. So far, this possibility was mostly investigated on large $\mathrm{Nd}$ :Glass lasers $^{2,3}$ that have a repetition rate of roughly one shot every 20 min. Even though PET isotopes were produced with activities larger than $10^{5} \mathrm{~Bq}^{2}$, their practical usage is limited due to this low repetition rate. A scheme based on existing, "table top," Ti:sapphire lasers, which typically operate at a repetition rate of $10 \mathrm{~Hz}$, would not suffer from this drawback. Compared to conventional accelerators, this would have a number of advantages: (i) the accelerating fields can exceed hundreds of $\mathrm{GV} / \mathrm{m}$, cutting down the acceleration length to some tens of microns; (ii) such laser systems are quite compact and cheap; and (iii) no shielding for radioprotection is needed up to the point where protons are generated. All this makes this approach potentially valuable, but it must be validated by the production of energetic and high-current proton beams with such laser facilities.

In this letter we report on an experiment during which we produced proton beams with an energy of up to $10 \mathrm{MeV}$ with a table top laser system, and show that our measurement, associated with kilohertz operation of such a laser, are consistent with a $\mathrm{GBq}$ source of ${ }^{11} \mathrm{C}$ or ${ }^{18} \mathrm{~F}$. We used the "Salle Jaune" laser at Laboratoire d'Optique Appliquée (LOA), operating at $10 \mathrm{~Hz}$ and a wavelength of $820 \mathrm{~nm}$ in the chiped pulse amplification (CPA) mode. It delivered on target energies of about $840 \mathrm{~mJ}$ in 40-fs full width at halfmaximum (FWHM) linearly polarized pulses, whose contrast ratio was better than $10^{-6} \cdot{ }^{4}$ Using a $f / 3$ off-axis parabolic mirror, the laser beam was focused at normal incidence onto the surface of thin foil targets. Since the focal spot had a waist of $4 \mu \mathrm{m}$, this resulted in peak intensities of up to 6 $\times 10^{19} \mathrm{~W} / \mathrm{cm}^{2}$. 


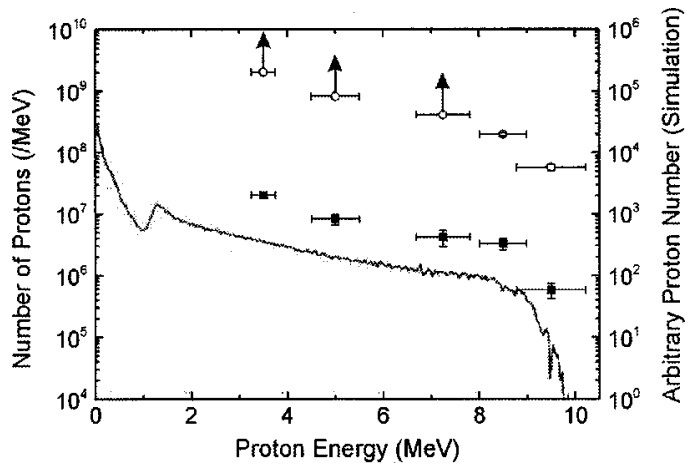

FIG. 1. Proton spectrum: Experimental measurement for the $6 \mu \mathrm{m}$ plastic (black dots) and aluminum target (light circles). For the latter, the arrows indicate the minimum number of protons, which would result in the saturation of the detectors. Note that the values from the two-dimensional PIC calculation are in arbitrary units.

CR-39 nuclear track detectors, which are sensitive to protons above $100 \mathrm{keV}$, were used to obtain the yield and opening cone of the proton beam. Protons hitting these detectors create pits, and the sampled number of these pits corresponds to the number of accelerated protons. The detectors were installed behind the target along its normal and were covered with aluminum foils, which served as energy filters. Changing the thickness of these filters, but keeping one area identical for comparison, this device gave the energy and the divergence of the proton beam simultaneously. The measured opening cone of the beam can be corrected with the well known Coulomb scattering within these foils. ${ }^{5}$ The energy loss of the protons is determined by the stopping power of aluminum. ${ }^{6}$

Figure 1 shows the measured proton energy distribution for $6-\mu \mathrm{m}$-thick plastic and aluminum foils. The energy of this beam reaches $10 \mathrm{MeV}$. As can be seen in Fig. 2, the proton beam is more collimated at higher energy. Recently, proton beams of only up to $1.5 \mathrm{MeV}$ have been reported with similar short-pulse and high-repetition-rate laser facilities, ${ }^{7}$ which is below most $Q$-values for PET. Protons up to 10 $\mathrm{MeV}$ have been observed with a $4 \mathrm{~J}$ laser ${ }^{8}$ however, the proton amount was not reported. This clearly stresses the importance of our result. Using 6- $\mu \mathrm{m}$-thick aluminum targets instead of plastic, the high-energy proton yield was enhanced by at least a factor of 100 . However, an accurate determination of the distribution below $8 \mathrm{MeV}$ could not be obtained due to this extremely high flux, which saturated the detectors. But the minimum value to obtain such a saturation can

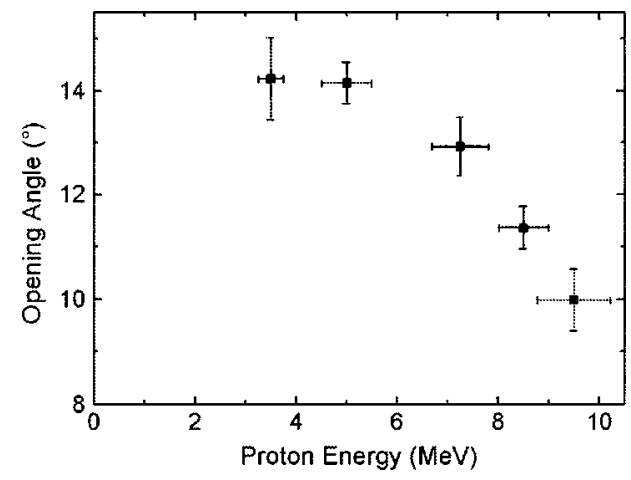

FIG. 2. FWHM of the proton beam shown in Fig. 1 obtained with the plastic target. be given by the minimum resolution of pits in the CR-39. The difference in the number of accelerated protons can be due to the different electrical response of the materials (isolator / conductor) or due to the initial condition of the preplasma. This subject is beyond the scope of the present work.

These measurements are in qualitative agreement with a kinetic simulation of this laser-plasma interaction that was performed with the particle-in-cell (PIC) code CALDER. In this calculation, a 35-fs laser pulse was focused at normal incidence onto a plastic target down to a $6.8 \mu \mathrm{m}$ FWHM spot, with a peak irradiance of $3 \times 10^{19} \mathrm{~W} / \mathrm{cm}^{2}$. The target was modeled as a $6 n_{c}$, fully ionized, $5 \mu \mathrm{m}$ thick carbon and hydrogen plasma, where $n_{c}=1.65 \times 10^{21} \mathrm{~cm}^{-3}$ is the critical density for a $820 \mathrm{~nm}$ laser. It is preceded by an exponential density gradient with a $4 \mu \mathrm{m}$ characteristic length, to account for the slight expansion that results from laser pre-pulses and amplified spontaneous emission. This small, lower density plasma is important as it favors an efficient energy transfer from the laser pulse to the target electrons. At the end of the pulse, almost $75 \%$ of the incident energy has been coupled to hot electrons that spread and form an electron cloud around the target. The electrons that leave the plasma and extend into vacuum set up a quasistatic electric field of the back of the target that reaches a maximum value of a few $\mathrm{TV} / \mathrm{m}$. As a result, proton and carbon ions are accelerated into vacuum from the rear side of the target, and the space-charge field decays as this expansion proceeds. Due to their lower mass, protons are accelerated with the highest efficiency. In less than $1 \mathrm{ps}$, the distributions of protons extends up to $10 \mathrm{MeV}$, as shown in Fig. 1. The regular variations, followed by an abrupt cutoff at the maximum energy, is similar to the experiment. The dip in the proton spectrum, observed around 1 $\mathrm{MeV}$, can be linked to the acceleration of carbon ions: as these ions are less mobile than protons, they are accelerated to a lower velocity, hence reducing the number of protons in the blow-off below that energy, compared to a pure proton expansion. Finally, we note that the smooth transverse variations of the accelerating field (with a computed FWHM of 16 $\mu \mathrm{m})$ explains why the proton beam is generated with a good collimation: the calculated angular FWHM at $(5 \pm 1) \mathrm{MeV}$ is $12^{\circ}$, in qualitative agreement with the experimental measurement.

The experimental proton energy spectra can be used to determine the expected activities of ${ }^{11} \mathrm{C}$ and ${ }^{18} \mathrm{~F}$ isotopes. We calculated the nuclear reaction yields with the CALDER MC code, using experimental values for the cross-sections ${ }^{9}$ and the Bethe formula for the stopping power. ${ }^{6}$ The ${ }^{11} \mathrm{~B}$ and ${ }^{18} \mathrm{O}$ targets modeled in the code were considered thick enough to slow down all incident protons below the respective reaction $Q$-values. This corresponds to an areal density of approximately $0.24 \mathrm{~g} / \mathrm{cm}^{2}$ for both targets. With the proton spectrum measured for the plastic targets, the calculated activities in ${ }^{11} \mathrm{C}$ and ${ }^{18} \mathrm{~F}$ samples are 12 and $2 \mathrm{~Bq}$ per shot, respectively. Accumulating laser shots at $10 \mathrm{~Hz}$ for $30 \mathrm{~min}$ with the same activation target, while taking into account the simultaneous isotope decay, results in activities of 134 and $29 \mathrm{kBq}$, respectively. Since for a given activation rate the decay matches production after a certain time, these integrated activities saturate at $209 \mathrm{kBq}$ for ${ }^{11} \mathrm{C}$ and $170 \mathrm{kBq}$ for ${ }^{18} \mathrm{~F}$. However, a typical patient dose for PET is $200 \mathrm{MBq}$, and it is necessary 
TABLE I. Calculated activities for the minimum proton beam obtained with the $6 \mu \mathrm{m}$ aluminum target. The secondary activation targets are chosen to have an areal thickness of $0.24 \mathrm{~g} / \mathrm{cm}^{2}$. The laser irradiation time would be $30 \mathrm{~min}$.

\begin{tabular}{cccc}
\hline \hline $\begin{array}{c}\text { Activation } \\
\text { target }\end{array}$ & $\begin{array}{c}Q \text {-value } \\
(\mathrm{MeV})\end{array}$ & $\begin{array}{c}\text { LOA laser } \\
\text { at } 10 \mathrm{~Hz} \\
\mathrm{MBq}(\mathrm{mCi})\end{array}$ & $\begin{array}{c}\text { LOA laser } \\
\text { at } 1 \mathrm{kHz} \\
\mathrm{MBq}(\mathrm{mCi})\end{array}$ \\
\hline${ }^{11} \mathrm{~B}$ & 2.76 & $13.4(0.36)$ & $1340(36.2)$ \\
${ }^{18} \mathrm{O}$ & 2.44 & $2.9(0.08)$ & $290(7.9)$ \\
\hline \hline
\end{tabular}

to go up to $800 \mathrm{MBq}$ so that fast chemistry can be performed to separate the tracer from the inactive carrier. ${ }^{2}$ It is thus impossible to get a medical dose with the current LOA setup. Thus, the flux of accelerated protons or the repetition rate of the laser needs to be increased. Performing the same calculation with the experimentally observed proton yield for aluminum targets instead of plastic, and increasing the repetition rate of the laser to $1 \mathrm{kHz}$, extends the 30 -min activities to the GBq order. As can be seen in Table I, this approach to use laser-plasma interactions hence appears comparable to the one using contemporary accelerators. There is also evidence that the efficiency of this scheme can be improved by operating at higher peak laser intensity. Calculations with CALDER and CALDER MC indicate that a modest increase in intensity to $8 \times 10^{19} \mathrm{~W} / \mathrm{cm}^{2}$, results in more protons at higher energies and leads to a sevenfold increase in ${ }^{18} \mathrm{~F}$ activity. This favorable intensity scaling is supported by recent experimental observations at $5 \times 10^{20} \mathrm{~W} / \mathrm{cm}^{2}$ which measured $3 \mathrm{MBq}$ of ${ }^{11} \mathrm{C}$, ${ }^{10}$ more than one order of magnitude greater than what was obtained at $5 \times 10^{19} \mathrm{~W} / \mathrm{cm}^{2}$ with the same experimental setup. ${ }^{2}$
In conclusion, our observations and calculations support the prospect of producing $\mathrm{GBq}$ sources of PET isotopes in the near future with table-top laser systems. We conservatively estimate that this goal could be met by a system delivering 1-J pulses focused to $10^{20} \mathrm{~W} / \mathrm{cm}^{2}$, and operating at $1 \mathrm{kHz}$.

One of the authors (P.McK.) is supported by a Royal Society of Edinburgh/SEELLD research fellowship.

${ }^{1}$ E. L. Clark, K. Krushelnick, J. R. Davies, M. Zepf, M. Tatarakis, F. N. Beg, A. Machacek, P. A. Norreys, M. I. K. Santala, I. Watts, and A. E. Dangor, Phys. Rev. Lett. 84, 670 (2000); R. A. Snavely, M. H. Key, S. P. Hatchett, T. E. Cowan, M. Roth, T. W. Phillips, M. A. Stoyer, E. A. Henry, C. Sangster, M. S. Sigh, S. Wilks, A. J. Mackinnon, A. A. Offenberger, D. M. Pennington, K. Yasuike, A. B. Langdon, B. F. Lasinski, J. Johnson, M. D. Perry, and E. M. Campbell, ibid. 85, 2945 (2000).

${ }^{2}$ I. Spencer, K. W. D. Ledingham, R. P. Singhal, T. McCanny, P. Mckenna, E. L. Clark, K. Krushelnick, M. Zepf, F. N. Beg, M. Tatarakis, A. E. Dangor, P. A. Norreys, R. J. Clarke, R. M. Allott, and I. N. Ross, Nucl. Instrum. Methods Phys. Res. B 183, 449 (2001).

${ }^{3}$ M. I. K. Santala, M. Zepf, F. N. Beg, E. L. Clark, A. E. Dangor, K. Krushelnick, M. Tatarakis, I. Watts, K. W. D. Ledingham, T. McCanny, I. Spencer, A. C. Machacek, R. Allot, R. J. Clarke, and P. A. Norreys, Appl. Phys. Lett. 78, 19 (2001).

${ }^{4}$ M. Pittman, S. Ferré, J. P. Rousseau, L. Notebeart, J. P. Chambaret, and G. Chériaux, Appl. Phys. B: Lasers Opt. 74, 529 (2002).

${ }^{5}$ R. M. Barnett, C. D. Carone, D. E. Groom, T. G. Trippe, C. G. Wohl, B. Armstrong, P. S. Gee, and G. S. Wagman, Phys. Rev. D 54, 1 (1996).

${ }^{6}$ T. A. Mehlhorn, J. Appl. Phys. 52, 6522 (1981).

${ }^{7}$ I. Spencer, K. W. D. Ledingham, P. McKenna, T. McCanny, R. P. S. Singhal, P. S. Foster, D. Neely, A. J. Langley, E. J. Divall, C. J. Hooker, R. J. Clarke, P. A. Norreys, E. L. Clark, K. Krushelnick, and J. R. Davies, Phys. Rev. E 67, 046402 (2003).

${ }^{8}$ K. Nemoto, A. Maksimchuk, S. Banerjee, K. Flippo, G. Mourou, D. Umstadter, and V. Yu. Bychenkov, Appl. Phys. Lett. 78, 595 (2001).

${ }^{9}$ EXFOR, http://dbd.nst.pku.edu.cn/exfor

${ }^{10} \mathrm{~K}$. W. D. Ledingham (private communication). 